\title{
Negative Dynamic Resistance and RF Amplification in Magnetic Tunnel Junctions
}

\author{
Hiroyuki Tomita $^{1 *}$, Hiroki Maehara ${ }^{2}$, Takayuki Nozaki ${ }^{1}$, and Yoshishige Suzuki ${ }^{1}$ \\ ${ }^{1}$ Graduate School of Engineering Science, Osaka University, 1-3 Machikaneyama, Toyonaka 560-8531, Japan \\ ${ }^{2}$ Electric Devices Engineering Headquarters, Canon ANELVA Corp, Kawasaki, Kanagawa 215-8550, Japan
}

(Received 20 January 2011, Received in final form 19 March 2011, Accepted 24 April 2011)

\begin{abstract}
We report on a numerical calculation study of two new functional properties in magnetic tunnel junctions (MTJs), negative dynamic resistance and RF amplification. The magnetic dynamics in a conventional $\mathrm{CoFeB} /$ $\mathrm{MgO/CoFeB} \mathrm{MTJ}$ with in-plane magnetization was investigated using a macro-spin model simulation. To examine the influence of thermal fluctuations, random external magnetic fields were also included. Using a voltage controlled bias circuit, the negative dynamic resistance was obtained from time averaged $I-V$ characteristics at both $0 \mathrm{~K}$ and $300 \mathrm{~K}$ under appropriate external magnetic fields and bias voltages. Using this negative dynamic resistance property, we demonstrated RF amplification with a $100 \mathrm{MHz}$ high frequency signal. Sizable RF amplification gain was observed without thermal fluctuation. However, at $300 \mathrm{~K}$, the RF signal was not amplified because low frequency magnetization dynamics were dominant.
\end{abstract}

Keywords : negative resistance, magnetic tunnel junction, amplification, spintronics

\section{Introduction}

Magnetic tunnel junction [1-4] (MTJ) devices have received much attention due to the ever-rising large tunnel magneto resistance (TMR) ratio. The large TMR ratio leads to large output signals in spin transfer torque $[5,6]$ phenomena. One of the most important applications is a magnetic random access memory with spin transfer switching [7-9], called the spin-RAM. Additionally, the spin transfer induced auto oscillator [10-12] and spin torque diode effect [13-16] have also been focused on. In this study, we used a macro-spin model simulation to investigate two new functional properties, negative dynamic resistance and RF amplification in MTJs.

\section{The Mechanism of Negative Dynamic Resistance and Amplification}

Negative dynamic resistance is a phenomenon whereby the current amplitude falls against an increase in bias voltage $(\mathrm{d} V / \mathrm{d} I<0)$. This property is well known in semiconductor devices, e.g. Esaki diode. We can expect a similar function in an MTJ device using the spin-transfer effect. The usual spin valve type MR device has two static

*Corresponding author: Tel: +81-6-6850-6425

Fax: +81-6-6850-6425, e-mail: hiro.tomita@spin.mp.es.osaka-u.ac.jp resistance states, $R_{\mathrm{P}}$ or $R_{\mathrm{AP}}$ depending on the parallel (P) or anti-parallel (AP) configuration of the free and reference layers. The spin transfer switching phenomenon without an external magnetic field excites a drastic change in resistance. For the negative dynamic resistance effect, we need to realize a continuous resistance change between the $\mathrm{P}$ and AP states. With an external magnetic field and spin transfer torque, we can excite spin torque auto oscillations and this gives an intermediate resistance between the P and AP states. Another important point is the measurement bias mode, i.e. voltage driven or current driven mode. In the MR device, these two modes show different $I-V$ curves. Images of the $I-V$ characteristics under the two bias modes can be seen in Fig. 1(a). In the voltage driven mode, we apply the voltage and measure the current. From the definition of negative dynamic resistance, the voltage variation $(\mathrm{d} V)$ divided by the current variation $(\mathrm{d} l)$ should be negative $(\mathrm{d} V / \mathrm{d} I<0)$. In the voltage driven case, with increasing bias voltage $(\mathrm{d} V>0), \mathrm{d} I$ should be negative for negative dynamic resistance. This means that the resistance should increase with increasing voltage. Voltage driven negative dynamic resistance can be observed in the transition from $\mathrm{P}$ to AP with increasing bias voltage. In the current driven mode with current stepping up $(\mathrm{d} I>0), \mathrm{d} V$ should be negative for negative resistance. This means that the resistance should reduce with increasing current. But in this bias direction we 
cannot excite the transition from AP to P. The situation is the opposite in the negative bias region.

Here, we introduce an electrical circuit for the RF amplification induced by the negative dynamic resistance property (see Fig. 1(b)). For our simulation, the dc bias voltage $\left(V_{\mathrm{dc}}\right)$ and RF signal $\left(V_{\mathrm{in}}(f)\right.$, frequency: $\left.f\right)$ are applied to the TMR device $\left(R_{\mathrm{TMR}}\right)$ and a series load resistance $\left(R_{\text {load }}\right)$. The amplified RF signal $\left(V_{\text {out }}(f)\right)$ is taken from the load resistance. The definition of amplification gain is as follows,

$$
\text { Gain }=V_{\text {out }}(f) / V_{\text {in }}(f)=\frac{R_{\text {load }}}{R_{\text {load }}+R_{\mathrm{TMR}}}
$$

With negative dynamic resistance $\left(R_{\mathrm{TMR}}<0\right)$, the gain becomes larger than 1 .

\section{Calculation Method}

In the numerical simulation, we assumed a conventional $\mathrm{CoFeB} / \mathrm{MgO} / \mathrm{CoFeB} \mathrm{MTJ}$. The reference layer magnetization direction is fixed. We simulated the magnetization dynamics of the free layer using the LLG equation with a spin transfer torque term in the macro spin model. The equation used is as follows,

$\frac{d}{d t} \vec{s}_{2}=\vec{s}_{2} \times \gamma \vec{H}_{e f f}-\alpha \vec{s}_{2} \times \frac{d}{d t} \vec{s}_{2}+g(\theta) \frac{I}{-e} \frac{\hbar / 2}{S} \vec{s}_{2} \times\left(\vec{s}_{1} \times \vec{s}_{2}\right)$

In this equation, $s_{1}\left(s_{2}\right), \gamma, H_{\mathrm{eff}}, \alpha, g(\theta)$ and $S$ are the unit spin angular momentum vector of the reference (free) layer, the gyro-magnetic constant, the effective magnetic field, the damping constant, the spin transfer torque efficiency [17] and the total spin angular momentum of the free layer, respectively. $H_{\text {eff }}$ includes an in-plane external magnetic field $\left(H_{\text {ext }}\right)$, an in plane magnetic anisotropic field $\left(H_{\mathrm{c}}\right)$, and a demagnetization field. In our configuration, $H_{\text {eff }}=\left(H_{\text {ext }}, H_{\mathrm{c}} y, M_{\mathrm{s}} z\right) ;\left(s_{2}=(x, y, z), M_{\mathrm{s}}\right.$ is the saturation magnetization). Additionally, in this study, we treated the thermal effect as a random magnetic field $\left(H_{\mathrm{r}}\right)[18,19]$. The amplitude of $H_{\mathrm{r}}$ is as follows,

$$
H_{r}=\sqrt{2 \alpha k_{B} T / \mu_{0} V M_{s} \gamma\left(1+\alpha^{2}\right) \Delta t}
$$

$k_{\mathrm{B}}, T, \mu_{0}, V$ and $\Delta t$ are the Boltzmann constant, the temperature, the magnetic permeability, the total volume of the magnetic free layer and the time step in the calculation, respectively. In our situation, $\alpha$ is small, so we approximated $\alpha^{2}$ equal 0.

\section{Results and Discussion}

The parameters used in this study are as follows. For the magnetic free layer, $M_{\mathrm{s}}=1000 \mathrm{emu} / \mathrm{cc}$, volume $V=2$ $\times 10^{-23} \mathrm{~m}^{3}, \alpha=0.01, H_{\mathrm{c}}=40$ Oe. For the properties of the MTJ, resistances $R_{\mathrm{P}}=400 \Omega$ and $R_{\mathrm{AP}}=1200 \Omega$, indicating a TMR ratio of $200 \%$. The calculated $R-H$ curves are shown in Fig. 2(a) under the different temperature conditions of $0 \mathrm{~K}$ (solid line) and $300 \mathrm{~K}$ (dotted line). For calculating the negative dynamic resistance and amplification properties, we used the voltage driven mode. The spin transfer torque, which is generated by a positive bias voltage, is specified to prefer the transition from the $\mathrm{P}$ to AP state in this study. For the simulation of the $I-V_{\mathrm{dc}}$ curves, we set the initial state $\mathrm{P}$ configuration and applied voltages from 0 to 1 V. Fig. 2(b) $(0 \mathrm{~K})$ and (c) $(300 \mathrm{~K})$ show $I-V$ curves with external magnetic fields of 0,30 ,

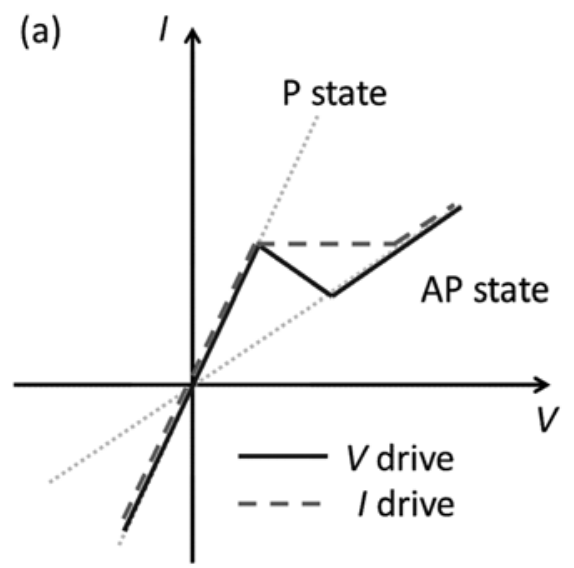

(b)

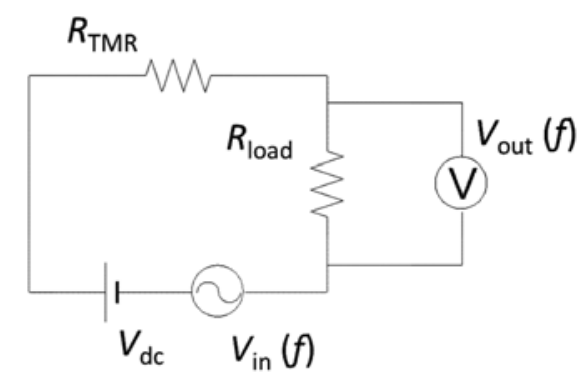

Fig. 1. (a) Concept of negative dynamic resistance in the $I-V$ curves of a MTJ under an external magnetic field. The black and gray curves show voltage driven and current driven $I-V$ curves transitioning from the $\mathrm{P}$ to the AP state, respectively. (b) Electric circuit for RF amplification with the MTJ. DC bias voltage $\left(V_{\mathrm{dc}}\right)$ and RF input signal $\left(V_{\mathrm{in}}(f)\right)$ are applied to a MTJ $\left(R_{\mathrm{TMR}}\right)$ and load resistance $\left(R_{\text {load }}\right)$ connected in series. The amplified output signal $\left(V_{\text {out }}(f)\right)$ is taken from $R_{\text {load. }}$. 
(a)

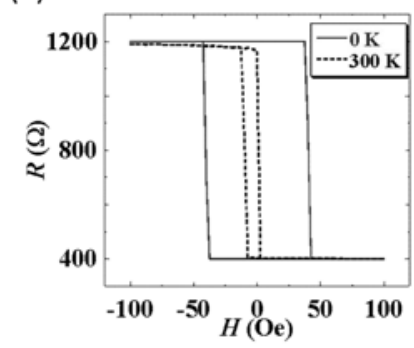

(b)

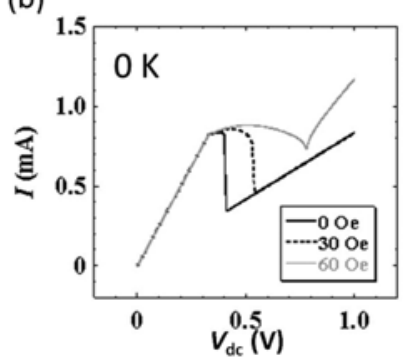

(c)

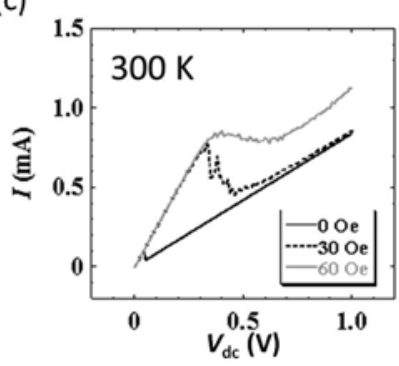

Fig. 2. Calculation results of (a) MR curves (solid line: $0 \mathrm{~K}$, dotted line: $300 \mathrm{~K}$ ) and voltage driven time averaged $I-V_{\mathrm{dc}}$ curves under various external magnetic fields at (b) $0 \mathrm{~K}$ and (c) $300 \mathrm{~K}$. Initial magnetization configuration is the $\mathrm{P}$ state for the $I-V_{\mathrm{dc}}$ curve calculations.

60 and 90 Oe, respectively. With the external magnetic fields, we were able to obtain intermediate resistance states and negative dynamic resistances both under $0 \mathrm{~K}$ and $300 \mathrm{~K}$ temperature conditions. In the calculation of the $I-V$ characteristics, the current value corresponds to the average value over $1 \mu \mathrm{s}$, which corresponds to a bandwidth of $0.5 \mathrm{MHz}$.

Next, we discuss the RF amplification property induced by the observed negative dynamic resistance. We simulated amplification of a $100 \mathrm{MHz}$ signal in the electrical circuit shown in Fig. 1(b). For the amplification, we applied a dc bias voltage from 0 to $2 \mathrm{~V}$ and an RF input signal $\left(V_{\text {in }}(f)=0.1 \mathrm{mV}, f=100 \mathrm{MHz}\right)$ to the MTJ with a load resistance $\left(R_{\text {load }}=780 \Omega\right)$ under an external magnetic field of 30 Oe. With 60 Oe external magnetic field, it also showed negative resistance. But with large current, it showed out-of plane precession. So in this time we mentioned 30 Oe condition. The initial magnetic configuration was the $\mathrm{P}$ state. In this condition, we monitored the output voltage signal $\left(V_{\text {out }}(f=100 \mathrm{MHz})\right)$ at the load resistance and evaluated the gain spectra. Fig. 3(a) shows the result of $V_{\text {out }}$ as a function of $V_{\mathrm{dc}}$ at $0 \mathrm{~K}$. An expanded image of the spectrum close to the peak structure is also shown in the inset. Here, $V_{\mathrm{dc}}$ is the total voltage applied to the $R_{\mathrm{TMR}}$ and $R_{\text {load. }}$. In the low bias voltage area $\left(V_{\mathrm{dc}}<0.5\right.$ $\mathrm{V})$, free layer magnetization is stabilized in the $\mathrm{P}$ state and the gain is less than 1 . By increasing $V_{\mathrm{dc}}$ to more than $1.0 \mathrm{~V}$, however, we were able to obtain a finite RF gain and the maximum $V_{\text {out }}(100 \mathrm{MHz})$ reached $0.6 \mathrm{mV}$, indicating a RF gain of 6 at $V_{\mathrm{dc}}=1.09 \mathrm{~V}$. In a higher bias voltage range, the magnetization configuration transits to the AP state, then the RF gain becomes less than 1 again.

Fig. 3(b) shows the calculated amplification property including the thermal noise effect $(300 \mathrm{~K})$. The parameters used are the same as in the previous calculation. $V_{\text {out }}(100 \mathrm{MHz})$ exceeds $10 \mathrm{mV}$ at the peak, which corresponds to a gain of 100 by our definition. However, since $V_{\text {out }}$ was observed even if the RF input power was zero, (a)

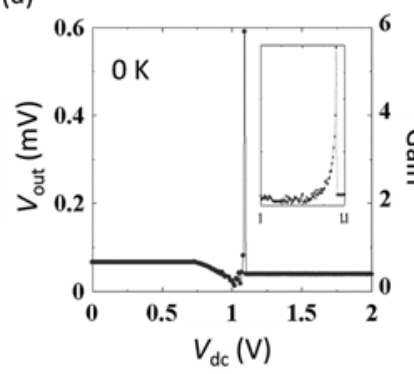

(b)

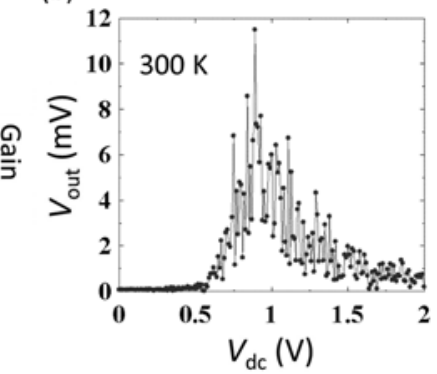

Fig. 3. (a) Output RF voltage signal $\left(V_{\text {out }}(100 \mathrm{MHz})\right)$ as a function of the bias voltage at $0 \mathrm{~K}$. The applied RF input signal $\left(V_{\text {in }}: 100 \mathrm{MHz}\right), R_{\text {load }}$ and the external magnetic field are $0.1 \mathrm{mV}, 780 \Omega$ and $30 \mathrm{Oe}$, respectively. The Gain value is defined as $V_{\text {out }} / V_{\text {in. }}$. The inset shows an expanded image around the amplification area $\left(V_{\mathrm{dc}} \sim 1 \mathrm{~V}\right)$. (b) $V_{\text {dc }}$ dependence of $V_{\text {out }}$ at $300 \mathrm{~K}$.

most of this signal may be due to noise. In this calculation, we averaged output voltage only $1 \mu$ s. To decrease noise level, we have to calculate longer time.

To understand the spin dynamics under RF amplification and the large output signal observed at $300 \mathrm{~K}$, we investigated the time domain output signal, and the FastFourier-Transform (FFT) spectrum. The conditions of the simulation are the same as used in the previous RF gain spectrum calculations. The input dc voltage was $1.09 \mathrm{~V}$ and $0.90 \mathrm{~V}$ for $0 \mathrm{~K}$ and $300 \mathrm{~K}$, respectively. Fig. 4(a) and (b) show the time domain $V_{\text {out }}$ signal and the FFT spectra at $0 \mathrm{~K}$. A clear oscillating output signal can be seen in the time domain signal. This produces clear peak structures in the FFT spectrum. The main peak at $0.95 \mathrm{GHz}$ corresponds to the spin torque oscillation signal. The peak appearing at $0.1 \mathrm{MHz}$ is the amplified signal. Side peaks are also observed around the main spin torque oscillation signal, at $0.95 \pm 0.1 \mathrm{GHz}$. These side peaks are generated by mixing of the signals between the spin torque oscillation $(f=0.95 \mathrm{GHz})$ and the input RF signal $(f=0.1$ GHz). Fig. 4(c) and (d) shows the calculation results of 
(a) $0 \mathrm{~K}$

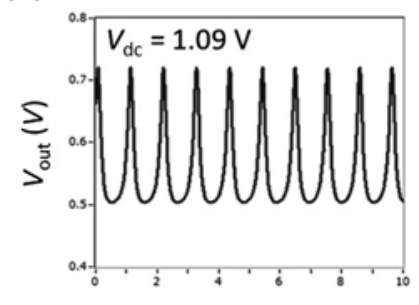

(c) $300 \mathrm{~K}$

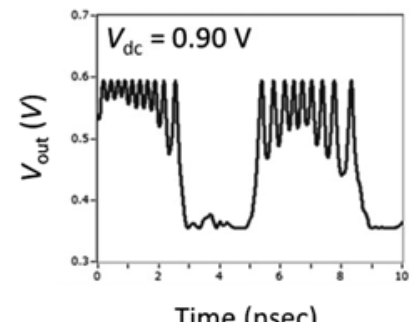

Time (nsec) (b) $0 \mathrm{~K}$

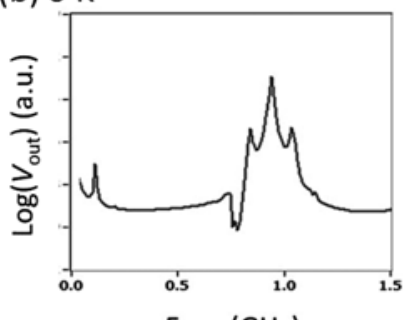

(d) $300 \mathrm{~K}$

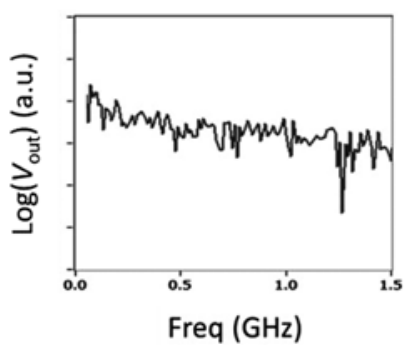

Fig. 4. Time domain output signal with (a) $V_{\mathrm{dc}}=1.09 \mathrm{~V}$ at $0 \mathrm{~K}$ and (b) $V_{\mathrm{dc}}=0.90 \mathrm{~V}$ at $300 \mathrm{~K}$. (c) and (d) show the FFT spectra of (a) and (b), respectively. The vertical axis is a logarithmic scale of the output signal voltage. The FFT spectra in (c) and (d) are on the same scale.

the time domain $V_{\text {out }}$ signal and the FFT spectra at $300 \mathrm{~K}$. Here we cannot see any clear stable oscillation. From the thermal fluctuations, the free layer magnetization switches between the $\mathrm{P}$ and AP states, the so called telegraph noise. Because of the high noise floor induced by this noise, the peak structure at $100 \mathrm{MHz}$ disappears and the magnetization dynamics at lower frequencies become dominant. To realize amplification of signal in this condition, we have to use lower frequency than telegraph noise dynamics.

In this simulation, we used a $100 \mathrm{MHz}$ signal, which corresponds to $1 / 10$ of that of the spin torque oscillation. By applying higher frequency signal, MR device cannot respond enough and amplification signal becomes smaller. And if input signal frequency becomes over the spin torque oscillation frequency, MR device is not responding anymore. For higher frequency signal amplification, we have to increase the frequency of the spin torque oscillation under the negative dynamic resistance condition. To realize this point, we have to excite ultra-high speed spin dynamics. Additionally, for the real application design for RF efficiency, for instance RF loss or impedance mismatch which were not treated in this simulation, is important.

\section{Conclusion}

In conclusion, the negative dynamic resistance and RF

amplification properties in an MTJ structure were investigated using a macro-spin model simulation including spin transfer torque and thermal effects. Under the optimum external magnetic field and application of a bias voltage, we were able to confirm a clear negative dynamic resistance effect in the time averaged $I-V$ curves at both $0 \mathrm{~K}$ and $300 \mathrm{~K}$. We also calculated the amplification property of the $100 \mathrm{MHz}$ signal induced by the negative dynamic resistance effect. With no thermal effect $(0 \mathrm{~K})$, we observed a sizable RF gain of 6 . At $300 \mathrm{~K}$, however, noise floor was high due to the telegraph noise. RF amplification would be obtained even at room temperature by optimizing the free layer's volume or magnetization, to increase thermal stability.

\section{Acknowledgements}

This work was partly supported by NEDO-"Spintronics Nonvolatile functional devices" project. H. Tomita is supported by Research Fellowship of the JSPS for Young Scientists.

\section{References}

[1] T. Miyazaki and N. Tezuka, J. Magn. Magn. Mater. 139, L231 (1995).

[2] J. S. Moodera, L. R. Kinder, T. M. Wong, and R. Meservey, Phys. Rev. Lett. 74, 3273 (1995).

[3] S. Yuasa, T. Nagahama, A. Fukushima, Y. Suzuki, and K. Ando, Nat. Mater. 3, 868 (2004).

[4] S. S. Parkin, C. Kaiser, A. Panchula, P. M. Rice, B. Hughes, M. Samant, and S.-H.Yang, Nat. Mater. 3, 862 (2004).

[5] J. C. Slonczewski, J. Magn. Magn. Mater. 159, L1 (1996).

[6] L. Berger, Phys. Rev. B 54, 9353 (1996).

[7] J. A. Katine, F. J. Albert, R. A. Buhrman E. B. Myers, and D. C. Ralph, Phys. Rev. Lett. 84, 3149 (2000).

[8] H. Kubota, A. Fukushima, Y. Ootani, S. Yuasa, K. Ando, H. Maehara, K. Tsunekawa, D. D. Djayaprawira, N. Watanabe, and Y. Suzuki, Jpn. J. Appl. Phys. 44, L1237 (2005).

[9] H. Tomita, K. Konishi, T. Nozaki, H. Kubota, A. Fukushima, K. Yakushiji, S. Yuasa, Y. Nakatani, T. Shinjo, M. Shiraishi, and Y. Suzuki, Appl. Phys. Express 1, 061303 (2008).

[10] E. B. Myers, D. C. Ralph, J. A. Katine, R. N. Louie, and R. A. Buhrman, Science 285, 867 (1999).

[11] S. I. Klselev, J. C. Sankey, I. N. Krivorotov, N. C. Emley, R. J. Schoelkopf, R. A. Buhrman, and D. C. Ralph, Nature 425, 380 (2003).

[12] A. M. Deac, A. Fukushima, H. Kubota, H. Maehara, Y. Suzuki, S. Yuasa, Y. Nagamine, K. Tsunekawa, D. D. Djayaprawira, and N. Watanabe, Nature Phys. 4, 803 (2008). 
[13] A. A. Tulapurkar, Y. Suzuki, A. Fukushima, H. Kubota, H. Maehara, K. Tsunekawa, D. D. Djayaprawira, N. Watanabe, and S. Yuasa, Nature 438, 339 (2005).

[14] J. C. Sankey, Y.-T. Cui, J. Z. Sun, J. C. Slonczewski, R. A. Buhrman, and D. C. Ralph, Nature Phys. 4, 67 (2008).

[15] H. Kubota, A. Fukushima, K. Yakushiji, T. Nagahama, S. Yuasa, K. Ando, H. Maehara, Y. Nagamine, K. Tsunekawa, D. D. Djayaprawira, N. Watanabe, and Y. Suzuki, Nature Phys. 4, 37 (2008).
[16] S. Ishibashi, T. Seki, T. Nozaki, H. Kubota, S. Yakata, A. Fukushima, S. Yuasa, H. Maehara, K. Tsunekawa, D. D. Djayaprawira, and Y. Suzuki, Appl. Phys. Express 3, 073001 (2010).

[17] J. C. Slonczewski, Phys. Rev. B 71, 024411 (2005).

[18] W. F. Brown, Jr. Phys Rev. 1301677 (1963).

[19] H. Akimoto, H. Kanai, Y. Uehara, T. Ishizuka, and S. Kameyama, J. Appl. Phys. 97, 10N705 (2005). 